\title{
The Effect of Rotating Collector Design on Tensile Properties and Morphology of Electrospun Polycaprolactone Fibres
}

\author{
Adhi Anindyajati ${ }^{a}$, Philip Boughton ${ }^{\mathrm{b}}$, and Andrew Ruys ${ }^{\mathrm{C}}$ \\ The University of Sydney, Australia
}

\begin{abstract}
Electrospinning is a technique that can produce fibres in the nanoscale range. This process is useful for many applications, including fabrication of fibrous scaffolds for fibrocartilage tissue engineering. For this application, cell attachment and tissue development is influenced by fibre morphology and mechanical properties. This electrospinning study investigated the influence of rotating collector design on morphology and mechanical properties of electrospun polycaprolactone fibre. The experiment employed 4 mandrel designs: 1) full surface of aluminium; 2) with gap feature; 3) with gap feature and teflon support; 4) with gap feature and tape support. The highest elastic modulus was obtained from mandrel with gap and tape support, which was $24.6 \mathrm{MPa}$ and significantly higher compared to fibres acquired from other collector designs. Fibre diameter attained was identical across the different collectors, ranging from $0.5-2 \mu \mathrm{m}$. Gap introduction showed enhanced alignment in the resultant fibre. It can be concluded that fibre alignment and tensile properties can be improved by simply modifying the collector design. This improved fibre mat can be developed as a biomaterial for fibrocartilage tissue engineering scaffolds.
\end{abstract}

\section{Introduction}

Electrospinning is a versatile and efficient method to produce fibre from submicron to nanoscale [1]. This technique can be applied in various materials and applications, including those in protective clothing, pharmaceutical, optical electronics, biosensors, environmental engineering, and tissue engineering [1-4]. The electrospinning process is driven by electrostatic force in the polymer solution. The electric field causes tensile force that stretches the polymer jet and drives it to a collector [3]. During travelling period, the solvent evaporates, leaving only stretched polymer which then deposits onto collector in fibre form. The advantage of electrospun fibres is mainly attributed to the characteristic of high surface area and interconnected pores [4].

Many devices applying nanofibrous structure require well aligned and ordered arrangement, such as those in microelectronics and photonics, nanoreinforcement, and tissue engineering [5-8]. Application of electrospun fibres in tissue engineering is strongly related to its potential in resembling the morphology of extracellular matrix in the body [9]. Tissue engineering itself is an approach of integrating engineering principles and life science to develop biological substitutes that restore, maintain, or improve tissue function [10]. A scaffold takes important part in this approach, providing temporary environment to allow tissue ingrowth. Therefore, a scaffold that featuring similar structure with corresponding tissue is desirable in order to promote neo-tissue formation analogous to that being substituted.

Tissues in musculoskletal system exhibit specific fibre alignment, mainly those that function as load bearing structures $[11,12]$. This system includes fibrocartilage, a connective tissue located in the joints, for instance glenoid labrum in shoulder, acetabular labrum in hip, annulus fibrossus in intervertebral joint, and meniscus in knee, as well as tendons and ligaments [1315]. Regarding the application of tissue engineering for fibrocartilage, fibre orientation of a scaffold is considered important, as it guides cell alignment and extracellular matrix deposition [12, 16]. Musculoskletal cells, for example fibroblasts of anterior cruciate ligament in the knee, tend to orientate along fibre direction, with those positioned within parallel fibre showing enhanced proliferation [16]. Furthermore, aligned fibres support an organized neo-tissue formation that yields stronger mechanical properties $[17,18]$.

A rotating mandrel of conductive material is commonly used as the electrospinning collector to achieve aligned fibre. High rotational speed is required to achieve a certain degree of alignment. Several attempts also have been proposed to improve fibre directionality, mainly related to the collector design. A wheel-like bobbin with tapered edge produced parallel arrays of nanofibres focused on the sharp edge of the wheel [5]. Using conductive plates with gap introduction, uniaxial fibres were formed in the gap region and oriented perpendicularly to the gap edges $[8,19]$. Mandrel from an insulated material and copper-wired drum were also able

Corresponding author: ${ }^{a}$ aani2456@uni.sydney.edu.au, ${ }^{b}$ philip.boughton@sydney.edu.au, ${ }^{c} \underline{\text { andrew.ruys@ } @ \text { sydney.edu.au }}$ 
to produce sub-micron fibre sheet with aligned structure $[7,20]$. Yarn with oriented and continuous microfibres has also been fabricated using a dual collection ring [21]. Aligned fibrous structure with improved mechanical properties was achieved using rotary jet spinning, in which both centrifugal and electrostatic force enhance polymer crystallinity and molecular orientation [22-24].

An aligned fibremat with sufficient length and thickness is needed for scaffold fabrication. This can be achieved by simply applying a rotating aluminium drum as the electrospinning collector. However, a very high rotation speed is required to achieve the desired alignment [16, 17]. In this study, an integration of a rotating mandrel collector with gap introduction is presented. Polycaprolactone (PCL) was used in this study, as this material is promising as a biodegradable scaffold for tissue engineering applications $[25,26]$. The effect of gap introduction on the mechanical properties and fibre morphology of the resulted fibre will be examined. Those properties will then be assessed regarding the potential for usage as a scaffold for fibrocartilage application.

\section{Materials and methods}

\subsection{Electrospinning}

Polycaprolactone (Mw 80.000) and acetone (Barnes, Australia) were used to make a polymer solution. The PCL solution was prepared by dissolving PCL polymer pellets in acetone overnight to create $10 \% \mathrm{w} / \mathrm{v}$ concentration. The collector designs were based on aluminium tubes (diameter $60 \mathrm{~mm}, 180 \mathrm{~mm}$ length), varied into four types: 1) solid surface of aluminium, 2) tube with gap feature, 3 ) solid surface of aluminium and Teflon, and 4) solid surface of aluminium and tape. Collector tubes 3 and 4 were basically collector 2 with the gap covered respectively by Teflon sheet and tape. The introduction of gap in the rotating collector was purposed to improve fibre alignment, while the use of those non-conductive covers was intended to support the deposited fibres in the gap area, considering the risk of fibre breakage due to electrostatic repulsion or overweight [19]. The tube with aluminium surface served as a control representing conventional drum collector, to compare the outcome of the newly-designed collector on fibre properties.

The electrospinning was set at a flow rate of $3 \mathrm{ml} / \mathrm{h}, 15$ $\mathrm{cm}$ working distance, mandrel rotation of 1000 RPM. A10 ml syringe with $20 \mathrm{G}$ needle was used to dispense the solution onto a grounded rotating collector. The needle tip was connected to a van der Graaf generator to charge the solution. Ejection rate was adjusted using syringe pump (Injectomat Tiva Agilia). Each sample was produced using approximately $2 \mathrm{ml}$ of PCL solution. Temperature around the syringe was controlled at $37^{\circ} \mathrm{C}$.

\subsection{Morphological analysis}

To investigate how different design types of rotating collector influencing fibre structures, SEM (Scanning Electron Microscopy - Zeiss EVO) was used to image morphological features of the samples, in terms of fibre diameter and alignment. 15 random measurements of the fibre diameter were performed for samples obtained from each collector using imageJ software.

\subsection{Tensile test}

Mechanical features of the samples were examined using a tensile test. 3 rectangular samples of $10 \times 80 \mathrm{~mm}$ were cut from a membrane produced by each collector types. The samples were tensile tested at room temperature, using an Instron 5567 Universal Testing Machine with $100 \mathrm{~N}$ load cell. To avoid slipping, sandpaper was applied in both ends of the specimens. Those samples were then clamped with the length between the clamps set at $60 \mathrm{~mm}$. Strain rate was adjusted to $50 \mathrm{~mm} /$ minute and preload was set to $0.2 \mathrm{~N}$. Young's modulus was calculated from the slope of the stress-strain curve in the linear region.

\subsection{Statistical analysis}

ANOVA and Tukey analysis were performed using Minitab 17 software, to analyse the difference of fibre properties obtained from different collector designs, in terms of fibre diameter, elastic modulus, maximum strength, and elongation at break.

\section{Results and discussion}

\subsection{Fibre diameter and morphology}

The electrospinning process produced a fibrous mat with an average thickness of $40 \mu \mathrm{m}$. Collector design 1, 2, 3, and 4 produced submicron fibres with diameter of $1.30 \pm 0.79 \mu \mathrm{m}, 1.24 \pm 0.75 \mu \mathrm{m}, 1.59 \pm 0.86 \mu \mathrm{m}, 1.78 \pm 1.07$ $\mu \mathrm{m}$, respectively (Fig. 1, Fig. 2). There is no noticeable difference in fibre diameter obtained from those different types of collector $(p>0.05)$. In the collector with gaps (design 2-4), fibres showed directionality, mainly those in the background or lower layer. On the other hand, fibres from the collector with full surface (design 1) exhibited a more random arrangement.

More aligned fibres formed in the gap area, which can possibly be attributed to the two sets of electrostatic forces that work in the charged fibres [19]. The first is a result of the electrostatic field and the second comes from Coulomb interactions between the positive charges in the nanofibres and the negative charges in conductive surfaces of the mandrel. In rotating collectors, those forces also work, as explained in the study of electrospinning using a wire drum collector [7]. During rotation, conductive surfaces of the mandrel alternately move closer to the needle tip. The electrospun fibre then follows the electric field generated by the first electrostatic force, which changes from one conductive surface to the next, creating fibre movement between the contiguous surfaces. The second electrostatic force then also took part, enhancing fibre stretching and alignment. In the collector with gap (design 2-4), entangled and 
unaligned fibres could also have formed, as the processing time increased. It is possibly caused by charge accumulation in the already deposited fibre, or that the collected mat acts as a conductive collector [7].

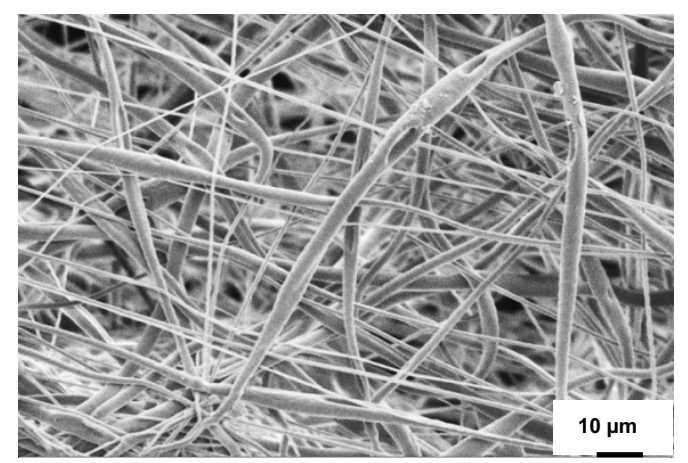

Collector design 1

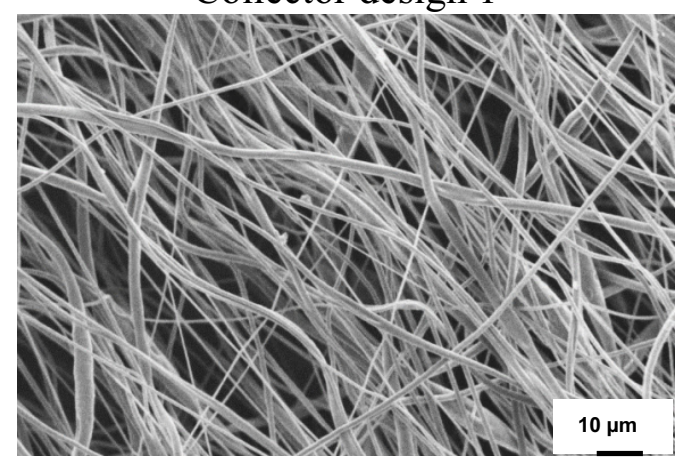

Collector design 3

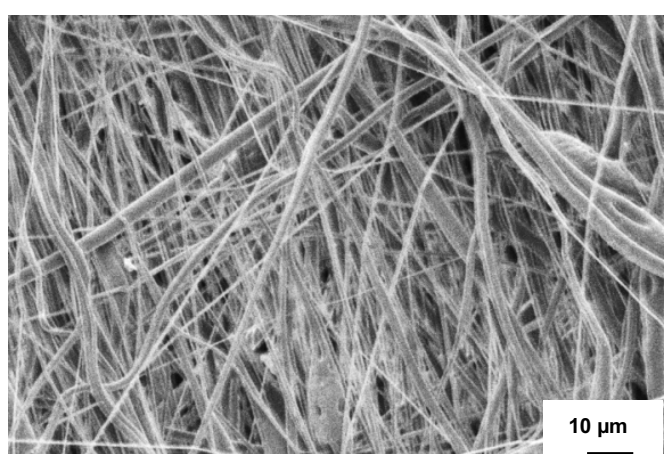

Collector design 2

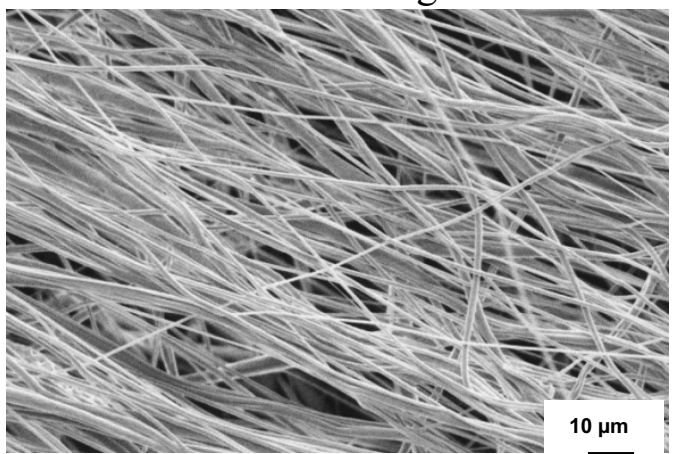

Collector design 4

Figure 1. Fibre morphology from different collector design

\subsection{Tensile properties}

Tensile properties of the fibres from different collector design are summarized in Fig. 2. Collector design 1, 2, 3, and 4 produced fibrous mat with respective elastic modulus of $14.21 \pm 2.66 \mathrm{MPa}, 12.03 \pm 1.10 \mathrm{MPa}, 8.32 \pm 4.30$ $\mathrm{MPa}, 24.60 \pm 4.18 \mathrm{MPa}$; maximum strength of $2.63 \pm 0.53$ $\mathrm{MPa}, 1.60 \pm 0.71 \mathrm{MPa}, 0.88 \pm 0.26 \mathrm{MPa}, 4.49 \pm 0.49 \mathrm{MPa}$; and elongation at break of $84.17 \pm 6.25 \%, 39.96 \pm 11.06 \%$, $25.56 \pm 3.86 \%, 67.78 \pm 8.66 \%$. Elastic modulus and maximum strength of the fibre from collector 4 is significantly higher $(p<0.05)$ compared to that collected from other collectors. Interestingly, fibre with the most elongation was obtained from collector 1 .

The higher value of fibre from collector 4 is possibly attributed to the stretching effect related to gap introduction. It has been reported that a gap in a charged collector induced polymer chain orientation within electrospun nanofiber [27]. Additionally, tensile force induced by rotating mandrel can also increase molecular alignment and crystallinity [28]. The improved elastic modulus achieved in this mandrel with gap may be strongly attributed to the enhanced molecular orientation and crystallinity, as demonstrated in the study of rotary jet electrospinning $[23,24]$. The tape covering the gap was also likely providing support for fibre deposition. However, the mechanism behind the low tensile properties in fibres from collector 2 and 3 is still unclear. There is probably structural breakage, due to fibre repulsion or the inability of the fibre to support its weight and tension [19]. In collector 3, the installed Teflon sheet on gap region also appeared to be unable to provide support. Teflon might resist fibre attachment and provide repulsion effect [29]. In contrast with collector 4, fibres from collector 1 might not undergo drawing and tension, thus providing a more elastic property.

Random fibre arrangement might contribute to the higher elongation value. It is also confirmed by other studies. Electrospinning using different mandrel rotation speeds $(0-8 \mathrm{~m} / \mathrm{s})$ showed that higher speeds produce fibres with higher alignment and tensile moduli, but yield lower ultimate strains [11]. One possible explanation is that it was due to fibre engagement at the beginning of deformation. Another observation also revealed that the increase in collector rotation speed $(0-1500$ RPM $)$ resulted in higher stiffness but lower strain [16]. 

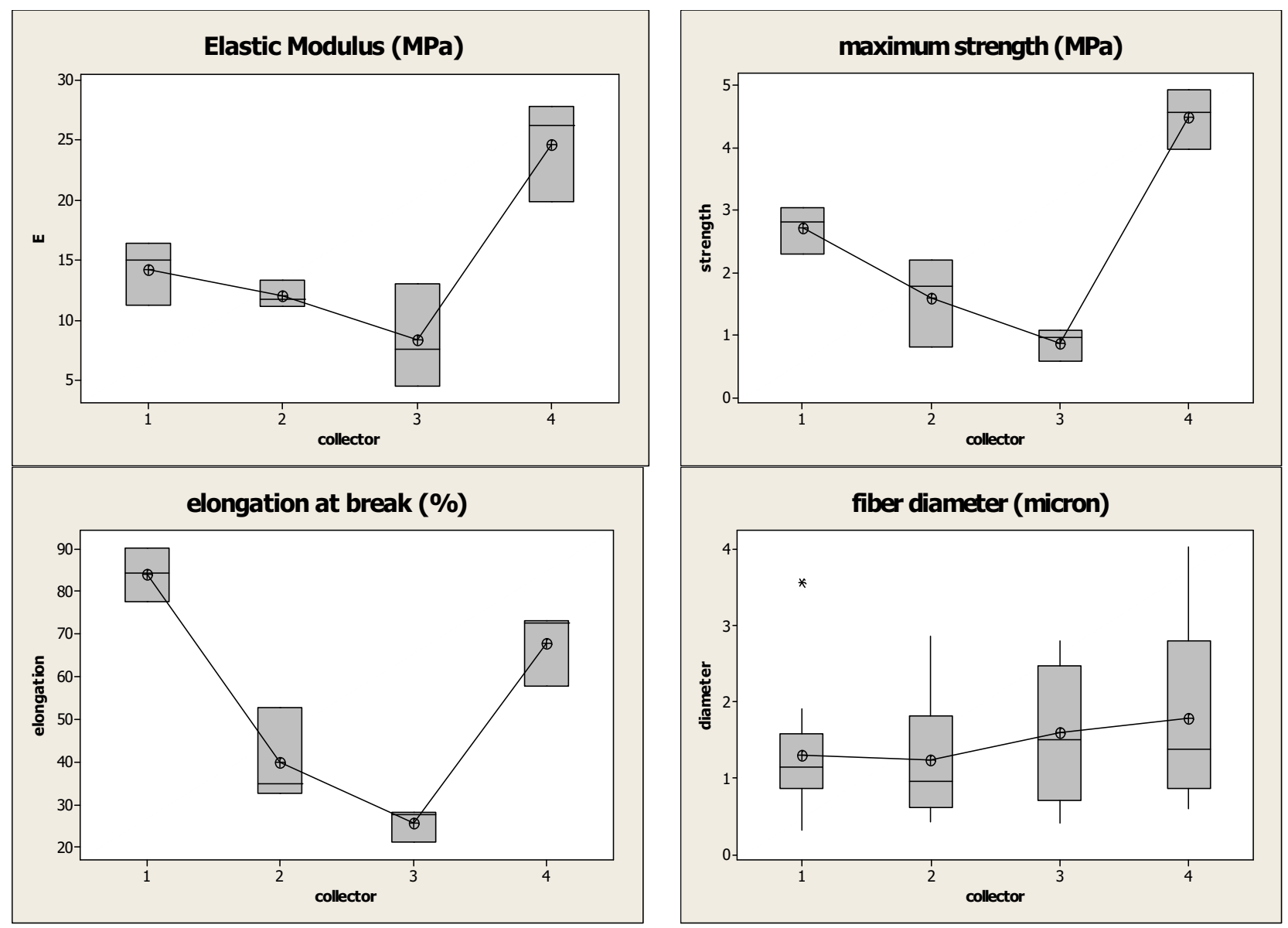

Figure 2.Tensile properties and diameter of fibres collected from different collector designs

\subsection{Potential application for tissue engineering scaffold}

Fibrocartilage has the structural and functional properties of fibrous connective tissue [14]. Its mechanical properties vary amongst different tissues, but in general, the tensile strength is about $10 \mathrm{MPa}$ and the elongation at rupture is about $13 \%$ [14]. In terms of those properties, fibres obtained in this study may be not compatible for general fibrocartilage. However, they are possibly compatible with acetabular labrum, a fibrocartilage tissue in the hip joint, which has an elastic modulus of about 25 $\mathrm{MPa}$ and elongation of about 50\% [30, 31].These values are similar to the properties of fibres obtained from collector 4. Labrum also possesses a multilayer structure of aligned and random collagen fibrils [32], thus the structure achieved in this study may potentially mimic the construct of labrum.

This experiment exhibited two type of fibrous structure with distinct characteristics: more elastic fibres with random alignment (collector 1) and stiffer fibres with more directionality (the other collectors). Previous studies suggested that cells appeared to favour a fibrous scaffold with a higher degree of alignment, in terms of cell alignment, proliferation, and extracellular matrix production $[11,16]$. However, less aligned fibre exhibited larger pore sizes, resulting in a higher number of cells attached, although in the longer term (7 and 14 days) the difference was not significant [16]. Therefore, further studies need to be conducted to study the relationship between fibre properties (mechanical and morphological) and cell behaviour.

\section{Conclusion}

Improvement in the alignment and tensile properties of electrospun fibre can be achieved by modifying the collector design. In this study, those improvements were achieved by applying mandrel with gaps as electrospinning collector. The resultant fibre possesses a structure with a multiple degree of alignment and tensile properties that is potentially suitable for fibrocartilage tissue engineering scaffold, specifically acetabular labrum. A scaffold with compatible morphology and mechanical characteristic can be expected to promote tissue ingrowth and healing. This construct can be further developed into a medical device applicable to aid recovery in hip injury correlated to tears in acetabular labrum. Further studies will address optimization of the electrospinning parameters, as well as its performance in in-vitro studies. 


\section{Acknowledgement}

The authors acknowledge the facilities and the scientific and technical assistance of the Australian Microscopy \& Microanalysis Research Facility at the Australian Centre for Microscopy \& Microanalysis at the University of Sydney.

\section{References}

1. Q. P. Pham, U. Sharma and A. G. Mikos, Electrospinning of Polymeric Nanofibers for Tissue Engineering Applications: A Review, Tissue Engineering 12 (2006) 1197-211.

2. H. W. Kim, H. E. Kim and J. C. Knowles, Production and Potential of Bioactive Glass Nanofibers as a Next-Generation Biomaterial, Advanced Functional Materials 16 (2006) 1529-1535.

3. D. H. Reneker and I. Chun, Nanometre diameter fibres of polymer, produced by electrospinning, Nanotechnology 7 (1996) 216-223.

4. N. Bhardwaj and S. C. Kundu, Electrospinning: A fascinating fiber fabrication technique, Biotechnology Advances 28 (2010) 325-347.

5. A. Theron, E. Zussman and A. Yarin, Electrostatic field-assisted alignment of electrospun nanofibres, Nanotechnology 12 (2001) 384-390.

6. C.-C. Liao, C.-C. Wang, K.-C. Shih and C.-Y. Chen, Electrospinning fabrication of partially crystalline bisphenol A polycarbonate nanofibers: Effects on conformation, crystallinity, and mechanical properties, European Polymer Journal 47 (2011) 911924.

7. P. Katta, M. Alessandro, R. D. Ramsier and G. G. Chase, Continuous Electrospinning of Aligned Polymer Nanofibers onto a Wire Drum Collector, Nano Letters 4 (2004) 2215-2218.

8. R. Dersch, T. Liu, A. K. Schaper, A. Greiner and J. H. Wendorff, Electrospun nanofibers: Internal structure and intrinsic orientation, Journal of Polymer Science Part A: Polymer Chemistry 41 (2003) 545-553.

9. A. Cipitria, A. Skelton, T. R. Dargaville, P. D. Dalton and D. W. Hutmacher, Design, fabrication and characterization of PCL electrospun scaffolds - A review, Journal of Materials Chemistry 21 (2011) 9419-9453.

10. R. Langer and J. P. Vacanti, Tissue engineering, Science 260 (1993) 920-6.

11. W.-J. Li, R. L. Mauck, J. A. Cooper, X. Yuan and R. S. Tuan, Engineering controllable anisotropy in electrospun biodegradable nanofibrous scaffolds for musculoskeletal tissue engineering, Journal of Biomechanics 40 (2007) 1686-1693.

12. N. L. Nerurkar, S. Sen, A. H. Huang, D. M. Elliott and R. L. Mauck, Engineered disc-like angle-ply structures for intervertebral disc replacement, Spine 35 (2010) 867-873.

13. R. M. Seldes, V. Tan, J. Hunt, M. Katz, R. Winiarsky and J. Robert H. Fitzgerald, Anatomy, Histologic Features, and Vascularity of the Adult Acetabular
Labrum, Clinical Orthopaedics and Related Research 382 (2001) 232-240.

14. M. Benjamin and E. J. Evans, Fibrocartilage, Journal of Anatomy 171 (1990) 1-15.

15. M. Benjamin and J. R. Ralphs, Fibrocartilage in tendons and ligaments - an adaptation to compressive load, Journal of Anatomy 193 (1998) 481-494.

16. L. Koepsell, T. Remund, J. Bao, D. Neufeld, H. Fong and Y. Deng, Tissue engineering of annulus fibrosus using electrospun fibrous scaffolds with aligned polycaprolactone fibers, Journal of Biomedical Materials Research Part A 99A (2011) 564-575.

17. B. M. Baker and R. L. Mauck, The effect of nanofiber alignment on the maturation of engineered meniscus constructs, Biomaterials 28 (2007) 1967-1977.

18. N. L. Nerurkar, W. Han, R. L. Mauck and D. M. Elliott, Homologous structure-function relationships between native fibrocartilage and tissue engineered from MSC-seeded nanofibrous scaffolds, Biomaterials 32 (2011) 461-468.

19. D. Li, Y. Wang and Y. Xia, Electrospinning of polymeric and ceramic nanofibers as uniaxially aligned arrays, Nano Letters 3 (2003) 1167-1171.

20. B. Sundaray, V. Subramanian, T. S. Natarajan, R.-Z. Xiang, C.-C. Chang and W.-S. Fann, Electrospinning of continuous aligned polymer fibers, Applied Physics Letters 84 (2004) 1222-1224.

21. P. D. Dalton, D. Klee and M. Möller, Electrospinning with dual collection rings, Polymer 46 (2005) 611614.

22. M. R. Badrossamay, H. A. McIlwee, J. A. Goss and K. K. Parker, Nanofiber Assembly by Rotary JetSpinning, Nano Letters 10 (2010) 2257-2261.

23. C.-C. Liao, C.-C. Wang and C.-Y. Chen, Stretchinginduced crystallinity and orientation of polylactic acid nanofibers with improved mechanical properties using an electrically charged rotating viscoelastic jet, Polymer 52 (2011) 4303-4318.

24. C.-C. Liao, C.-C. Wang, C.-Y. Chen and W.-J. Lai, Stretching-induced orientation of polyacrylonitrile nanofibers by an electrically rotating viscoelastic jet for improving the mechanical properties, Polymer 52 (2011) 2263-2275.

25. D. Hutmacher, M. B. Hürzeler and H. Schliephake, A Review of Material Properties of Biodegradable and Bioresorbable Polymers and Devices for GTR and GBR Applications, International Journal of Oral and Maxillofacial Implants 11 (1996) 667-678.

26. M. A. Woodruff and D. W. Hutmacher, The return of a forgotten polymer-Polycaprolactone in the 21st century, Progress in Polymer Science 35 (2010) 1217-1256.

27. M. V. Kakade, S. Givens, K. Gardner, K. H. Lee, D. B. Chase and J. F. Rabolt, Electric Field Induced Orientation of Polymer Chains in Macroscopically Aligned Electrospun Polymer Nanofibers, Journal of the American Chemical Society 129 (2007) 27772782.

28. T. Kongkhlang, K. Tashiro, M. Kotaki and S. Chirachanchai, Electrospinning as a New Technique To Control the Crystal Morphology and Molecular Orientation of Polyoxymethylene Nanofibers, 
Journal of the American Chemical Society 130 (2008) 15460-15466.

29. A. Thorvaldsson, H. Stenhamre, P. Gatenholm and P. Walkenström, Electrospinning of Highly Porous Scaffolds for Cartilage Regeneration, Biomacromolecules 9 (2008) 1044-1049.

30. C. D. Smith, S. Masouros, A. M. Hill, A. A. Amis and A. M. J. Bull, A biomechanical basis for tears of the human acetabular labrum, British Journal of Sports Medicine 43 (2009) 574-578.
31. T. Ishiko, M. Naito and S. Moriyama, Tensile properties of the human acetabular labrum-the first report, Journal of Orthopaedic Research 23 (2005) 1448-53.

32. W. Petersen, F. Petersen and B. Tillmann, Structure and vascularization of the acetabular labrum with regard to the pathogenesis and healing of labral lesions, Archives of Orthopaedic and Trauma Surgery 123 (2003) 283-288. 